\title{
Chordal Axis on Weighted Distance Transforms
}

\author{
Jérôme Hulin and Edouard Thiel \\ Laboratoire d'Informatique Fondamentale de Marseille (LIF, UMR 6166), France \\ \{Jerome.Hulin, Edouard.Thiel\}@lif.univ-mrs.fr \\ http://www.lif.univ-mrs.fr/ ${ }^{\sim}$ thiel
}

\begin{abstract}
Chordal Axis (CA) is a new representation of planar shapes introduced by Prasad in 1, useful for skeleton computation, shape analysis, characterization and recognition. The CA is a subset of chord and center of discs tangent to the contour of a shape, derivated from Medial Axis (MA). Originally presented in a computational geometry approach, the CA was extracted on a constrained Delaunay triangulation of a discretely sampled contour of a shape. Since discrete distance transformations allow to efficiently compute the center of distance balls and detect discrete MA, we propose in this paper to redefine the CA in the discrete space, to extract on distance transforms in the case of chamfer norms, for which the geometry of balls is well-known, and to compare with MA.
\end{abstract}

Keywords: image analysis, shape description, chordal axis, medial axis, discrete geometry, chamfer or weighted distances.

\section{Introduction}

Shape description consists in extracting features from a binary image, like area, width, number of holes, etc. To this purpose, Blum first proposed the notion of Medial Axis (MA) of a shape $\mathcal{S}$ in [2. Then Pfaltz and Rosenfeld defined it in [3] as the set of centers of maximal discs in $\mathcal{S}$, a disc being maximal in $\mathcal{S}$ if it is not completely overlapped by any other disc included in $\mathcal{S}$. MA has become an important tool in image analysis and shape description, because it is a reversible coding and a global representation, centred in the shape, which allows to simplify, compress, or compute a skeleton of a shape.

Among several approaches in image analysis, we distinguish : the continuous approach with analytical pieces of curves, which is in general case difficult to tackle; the semi-continuous approach, which consists in sampling the contour of a shape, and then deals with computational geometry in Euclidean space, as Voronoï diagram, convex hull, etc; the discrete approach, which keeps the shape bitmap (or sample a continuous shape on the rectilinear grid) in discrete space $\mathbb{Z}^{n}$ and makes use of discrete geometry, often needing to redefine continuous properties in discrete ones.

MA has been studied in this three frameworks. A continuous MA is obtained in [4] with pieces of lines and arcs. The semi-continuous MA is an approximation of continuous MA, extracted from the Voronoï Diagram of the sampled contour 
(e.g., see [5]). The discrete MA is generally extracted by local tests on a Distance Transform (DT), which is an image where each shape point is labelled with its distance to the background.

Working on semi-continuous framework, Prasad proposed in [1] a new representation of shapes, called Chordal Axis (CA). The aim was to correct sensitiveness to noise in sampled contour for semi-continuous MA. The definition is close to MA, but has different properties. A Maximal Chord of Tangency (MCT) of a shape $\mathcal{S}$ is a chord of a maximal disc $D$ of $\mathcal{S}$, which separates the boundary of $D$ into two arcs such that one at least is not tangent to the boundary of $\mathcal{S}$; the $\mathrm{CA}$ is the set of midpoints of the MCTs, plus the set of centers of maximal discs having at least three MCTs. Prasad then extracted a semi-continuous CA from a Delaunay triangulation of sampled contour. In order to fix some zigzags in the result, Prasad introduced in [6] a valuation of certain internal edges, leading to a shape decomposition process.

We have recently proposed an algorithm for extracting discrete MA from a DT for any chamfer norm in [7] and for Squared Euclidean Distance in [8]. The algorithm computes a test neighbourhood and Look-Up Tables and then extracts the centers of maximal balls for the given distance by local tests on DT. In this paper we naturally propose to adapt the $\mathrm{CA}$ in discrete framework, in the case of $2 \mathrm{D}$ chamfer norms. A chamfer (or weighted) distance is an integer distance defined by a mask 9 ; such a distance allows very fast computation of DT with a sequential algorithm in two raster scans given in 10,11. We focus on the masks inducing a norm, for which the geometry of ball is established (a discrete convex polyhedron, see [12]).

We recall in Section 2 some basic notions and definitions. We present and justify our method in Section 3. Results are given in Section 4, and finally we conclude in Section 5 .

\section{Definitions}

\subsection{Chordal Axis}

Consider a shape $\mathcal{S}$ in $\mathbb{R}^{2}$ and $B$ a maximal ball in $\mathcal{S}$. Following Prasad in [1, a chord of $B$ is called a Maximal Chord of Tangency (MCT) if at least one of the arcs subtended by the chord is free of points of tangency with the boundary of $\mathcal{S}$. Fig. 1 gives some examples of MCTs.

The Chordal Axis (CA) of a shape is the set of all pairs $(p, \delta)$, where $p$ and $\delta$ are either the midpoint and half the length of a maximal chord of tangency, or the center and radius of a maximal ball which has at least three maximal chords of tangency. We call $\alpha$-points the midpoints of MCTs and $\beta$-points the centers of maximal balls having at least three MCTs.

In the continuous domain (see Fig. 1), the CA is generally non-connected, contrary to the MA. Indeed, the connectedness is broken as soon as a maximal disc inside a shape contains at least three maximal chords of tangency (MCTs). However, connectedness may easily be obtained if, in such maximal discs, we draw a segment from the center of the disc to the middle of each MCT. 


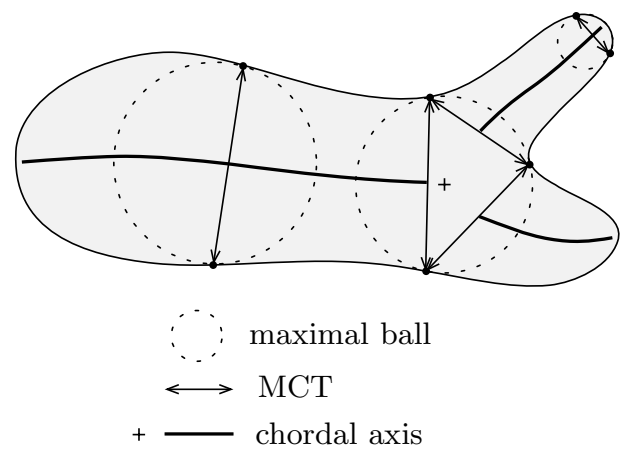

Fig. 1. Three maximal balls inside a shape and their MCTs; chordal axis

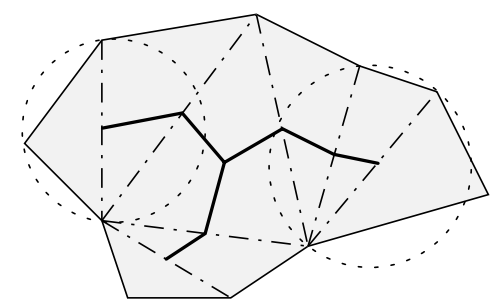

$\vdots \vdots$ circumcircle of Delaunay triangle

- internal edge of the triangulation

— CAT skeleton

Fig. 2. Delaunay triangulation of sampled contour and CAT skeleton

(c)
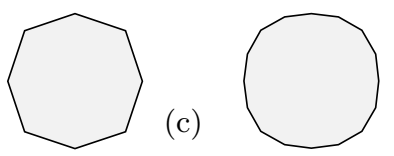

(c), $d_{\langle 5,7,11\rangle}$

In [1, Prasad defines the Chordal Axis Transform (CAT) as a semi-continuous method to extract the CA: he starts with a discrete sample of the boundary of a given shape $\mathcal{S}$, then calculates the Delaunay triangulation of these points, inside $\mathcal{S}$. The MCTs of $\mathcal{S}$ are approximated by the internal edges of the triangulation (the edges which are not on the boundary of $\mathcal{S}$ ). Then a skeleton based on the semi-continuous $\mathrm{CA}$ is constructed by connecting, inside each triangle, the midpoints of two or three internal edges, depending on the number of edges lying on the boundary of $\mathcal{S}$. The resulting skeleton (see Fig. 2) is sensitive to the irregularities of the samples of the contour of $\mathcal{S}$, giving to it angularities. In [6], a valuation on the edges of the triangulation is proposed in order to delete edges considered as weak and to smooth the skeleton; or to detect strong edges which split the object into significant parts, to achieve shape decomposition.

\subsection{Chamfer Distances and Norms}

Here we recall some results from [12]. A chamfer mask $\mathcal{M}$ in $\mathbb{Z}^{n}$ is a centralsymmetric set $\mathcal{M}=\left\{\left(\vec{v}_{i}, w_{i}\right) \in \mathbb{Z}^{n} \times \mathbb{Z}_{+*}\right\}_{1 \leqslant i \leqslant m}$ containing at least a basis of $\mathbb{Z}^{n}$, where $\left(\vec{v}_{i}, w_{i}\right)$ are called weightings, $\vec{v}_{i}$ vectors and $w_{i}$ weights. The chamfer distance $d_{\mathcal{M}}$ between two points $p, q \in \mathbb{Z}^{n}$ is

$$
d_{\mathcal{M}}(p, q)=\min \left\{\sum \lambda_{i} w_{i}: \sum \lambda_{i} \vec{v}_{i}=\overrightarrow{p q}, 1 \leqslant i \leqslant m, \lambda_{i} \in \mathbb{Z}_{+}\right\} ;
$$

it is shown that $d_{\mathcal{M}}$ is always a metric. A chamfer ball $B$ of center $p \in \mathbb{Z}^{n}$ and radius $R \in \mathbb{Z}_{+}$is $B_{\mathcal{M}}(p, R)=\left\{q \in \mathbb{Z}^{n}: d_{\mathcal{M}}(p, q) \leqslant R\right\}$. 
Consider $\mathcal{M}^{\prime}=\left\{O+\vec{v}_{i} / w_{i}\right\}_{1 \leqslant i \leqslant m} \in \mathbb{R}^{n}$ and let $B_{\mathcal{M}}^{\prime}=\operatorname{conv}\left(\mathcal{M}^{\prime}\right)$, then $B_{\mathcal{M}}^{\prime}$ is a central-symmetric and convex polyhedron whose facets separates $\mathbb{R}^{n}$ in cones from $O$. A facet $\mathcal{F}$ of $B_{\mathcal{M}}^{\prime}$ is generated by a subset $\left.\mathcal{M}\right|_{\mathcal{F}}=\left\{\left(\vec{v}_{j}, w_{j}\right)\right\}_{1 \leqslant j \leqslant n}$ of $\mathcal{M}$; if $\Delta_{\mathcal{F}}=\operatorname{det}\left\{\vec{v}_{j}\right\}_{1 \leqslant j \leqslant n}$ is such that $\left|\Delta_{\mathcal{F}}\right|=1$, then $\mathcal{F}$ is said unimodular. If each facet of $B_{\mathcal{M}}^{\prime}$ is unimodular, then $d_{\mathcal{M}}$ is a norm in $\mathbb{Z}^{n}$ (the converse is a difficult problem). Now let $d_{\mathcal{M}}$ be a chamfer norm, $\mathcal{F}$ a facet of $B_{\mathcal{M}}^{\prime}$ and $\left.\mathcal{M}\right|_{\mathcal{F}}=\left\{\left(\vec{v}_{j}, w_{j}\right)\right\}_{1 \leqslant j \leqslant n} ;$ then for any point $p=\left(y_{1}, \ldots, y_{n}\right)$ in the cone $(O, \mathcal{F})$ we have $d_{\mathcal{M}}(O, p)=y_{1} \delta_{1}+\cdots+y_{n} \delta_{n}$, where

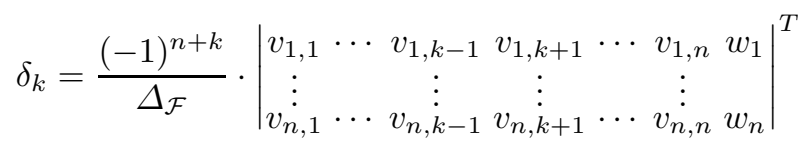

is the elementary displacement for coordinate $y_{k}$. Moreover, the chamfer ball $B$ has the same geometry as $B^{\prime}$ (up to a scale factor), so $\vec{\delta}_{\mathcal{F}}=\left(\delta_{1}, \ldots, \delta_{n}\right)$ is a normal vector of facet $\mathcal{F}$.

In $\mathbb{Z}^{2}$, a common way to denote small masks is $\langle a, b\rangle=\{(1,0, a),(1,1, b)\}$ and $\langle a, b, c\rangle=\{(1,0, a),(1,1, b),(2,1, c)\}$. Widely used chamfer norms in image analysis are $d_{4}=\ell_{1}=d_{\langle 1,2\rangle}, d_{8}=\ell_{\infty}=d_{\langle 1,1\rangle}, d_{\langle 3,4\rangle}$ and $d_{\langle 5,7,11\rangle}$, see Fig. 3 .

\section{Discretization of Chordal Axis}

In the following we work in $\mathbb{Z}^{2}$ with a given chamfer norm $d_{\mathcal{M}}$. Let $\mathcal{S}$ be a shape, a point $p$ of $\mathcal{S}$ is called a boundary point if its distance to the complement of $\mathcal{S}$ equals the smallest weight in $\mathcal{M}$. Consider $B$ a ball of $d_{\mathcal{M}}$ included in $\mathcal{S}$. A point $p$ is called a tangency point between $B$ and $\mathcal{S}$ if $p$ belongs to $B$ and to the boundary of $\mathcal{S}$. A tangency zone is a maximal 8-connected set of tangency points. We exhibit three properties about tangency of discrete balls, then present our algorithms to generate the discrete CA. Our aim is to generate MCTs which are meaningful with respect to description and shape analysis.

\subsection{Point Threshold}

In the discrete domain (see Fig. 4. b), the local intersection between a maximal ball included in an object and the boundary of this object is seldom a unique point. Let $A$ and $B$ be two tangency points between a maximal ball and the boundary of a shape $\mathcal{S}$. The chord $[\mathrm{AB}]$ is maximal if one of the two $\operatorname{arcs} \widehat{A B}$ is free of points of tangency with the boundary of $\mathcal{S}$. In the continuous case (Fig. 4.a), we have a unique MCT $[A B]$. In the discrete case (Fig. 4.b), two MCTs $\left(\left[A_{1} B_{2}\right]\right.$ and $\left.\left[A_{2} B_{1}\right]\right)$ appear, because the intersections between the ball and the shape's boundary are not single points anymore. Nevertheless only one chord should characterize this ball, the extra chord being an artefact of the discretization. For a given tangency zone, we need to decide whether it is considered as a point or not. To this end we introduce a point threshold, denoted PTH (in pixels). We measure the farthest Euclidean distance $d$ between the tangency zone 


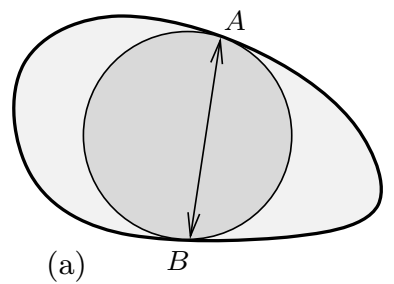

(a)

$\begin{array}{ll}\square \text { background } & \square \text { maximal ball } \\ \square \text { shape } & \square \text { center of maximal ball } \\ \longleftrightarrow \text { MCT } & \square \text { tangency point }\end{array}$

(b)
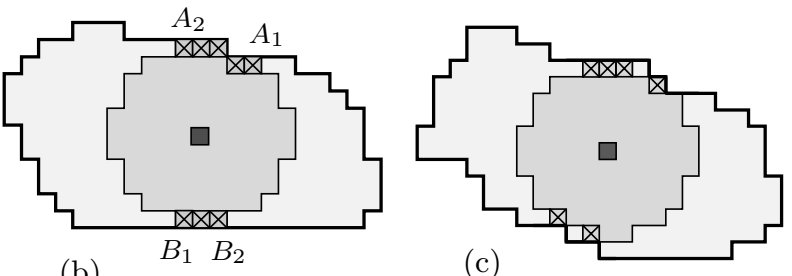

(c)

(d)

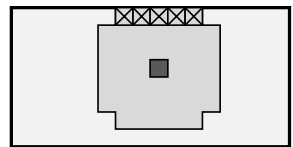

Fig. 4. Maximal Ball in continuous (a) and discrete (b),(c),(d) space

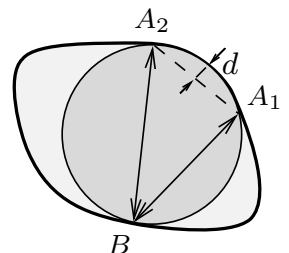

(a)

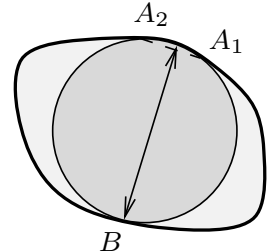

(b)

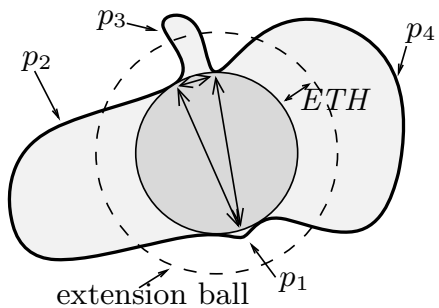

Fig. 5. Application of the point threshold

Fig. 6. Extension ball and protuberances on the boundary: neglected $\left(p_{1}\right)$ and non neglected $\left(p_{2}, p_{3}\right.$ and $\left.p_{4}\right)$; resulting MCTs

$\widehat{A_{1} A_{2}}$ and the chord $\left[A_{1} A_{2}\right]$ induced by the extremities of this zone (see Fig. 5 ). If $d$ is less then $P T H$, we contract the tangency zone to a single pixel, located in the middle of the zone (case (b): one MCT). Otherwise each extremity of the tangency zone becomes the endpoint of a MCT (case (a): two MCTs).

\subsection{Extension Threshold}

The local intersection between a maximal ball and the boundary of the shape may be composed of several non-connected components. E.g. in Fig. 4 (c), we observe that the discretization of the image splits each tangency zone into two connected components. The immediate construction of MCTs would lead to the appearance of two parasitic chords along the boundary of the object. Before proceeding with the construction of the MCTs, we must determine for each connected component, which tangency zone it should belong to. An appropriate solution consists in exploring the peripheral domain of the maximal ball, looking for a path in the boundary of the shape which connects some tangency components. 
We introduce an extension threshold, denoted ETH. Given a maximal ball $(O, R)$, we consider the extension ball $(O, R+E T H)$, see Fig. 6. A $n$-connected path from $p_{0}$ to $p_{k}$ is a sequence of points such that $p_{i}$ and $p_{i-1}$ are $n$-neighbours ( $n=4$ or $8,1 \leqslant i \leqslant k$ ); if there exists an 8 -connected path of points of the boundary of the object inside the extension ball which connects two (or more) tangency components, then we merge these components into a single one.

This peripheral search has another utility: it allows to ignore some noise features on the boundary of the shape. Indeed if a protuberance on the border is small enough to be fully included in the extension ball ( $p_{1}$ in Fig. 6), we will not generate any MCT at the base of this irregularity (unless the point threshold is exceeded). Therefore the value of the extension threshold has direct influence on the level of detail of our analysis. The higher the extension threshold is set to, the less precise this analysis near the boundary of the shape.

\subsection{Radius Increment}

In the discrete space, a maximal ball included in an object may not yield any MCT, as shown in Fig. 4 (d): several points belong to the boundary but our point threshold reduces the tangency zone to a unique point (there may even be a single tangency point in some configurations). This phenomenon does not question our point threshold (which avoids the creation of a parasitic MCT along the boundary of the object); it is only due to the fact that the working domain is the discrete grid. The most simple example is the case of an object having an horizontal or vertical branch with an even width. In this case the maximal balls inside this branch will only be tangent to one side of the shape (the chamfer balls are central-symmetrical so their diameter, measured horizontally in pixels, is odd). No MCT would then appear in such a branch.

We propose to increment the radius of maximal balls with a certain value $R I$ (Radius Increment), before proceeding with the exploration of their boundaries. Let $a$ be the smallest weight of the chamfer mask. Then $a$ equals the distance between two 4-neighbours, and it is sufficient to take $R I=a$ to make certain that all maximal balls have at least two tangency zones with the boundary of the shape. However, in most cases, we observe that an increment of value 1 is enough to ensure that almost all balls have two tangency zones.

\subsection{Algorithms to Generate the Discrete Chordal Axis}

The three proposed thresholds are independant and in practice, they enable to generate almost all useful MCTs, and avoid the creation of parasitic chords (lying along the boundary of the shape or describing noise features of the border). These thresholds are computed sequentially for each maximal ball, as follows:

1. calculate the new radius of the ball, by adding $R I$,

2. look for tangency zones in the peripheral region, using $E T H$,

3. decide for each tangency zone, if it should be contracted to a single point, using PTH. 
We recall (see [7]) that on DT, the value $D T(p)$ for any shape point $p$ is the radius of the greatest set $\left\{q \in \mathbb{Z}^{2}: D T(p)-d_{\mathcal{M}}(p, q)>0\right\}$ so the greatest ball centred in $p$ inside the shape is $\left\{q \in \mathbb{Z}^{2}: d_{\mathcal{M}}(p, q) \leqslant D T(p)-1\right\}$ which has radius $D T(p)-1$ (utilized line 4 in the procedure Gen_CA).

The boundaries of maximal balls are inspected thanks to the equations given in Section 2.2, or by consulting an image containing the distance values from any point to the top-left point of the image (computed once), then using symmetries.

Input $\quad I N$ Shape image, DT Distance Transform, $M A$ Medial Axis Output $C A$ Chordal Axis

Procedure Gen_CA (IN, DT, MA, CA, PTH, ETH, RI)

1 Initialize $C A$ to $\emptyset$

2 For all points $p$ in $I N$ do

$3 \quad$ If $p \in M A$ then

Procedure Search_MCTs $(p, R, D T, C A,\{$ Thresholds $\})$

1 IMAGE tmp //image storing tangency points, initialized to 0

2 INT $n b_{\text {tang }}=0 \quad / /$ number of tangency zones

3 Generate $\mathcal{C}$, the boundary of the ball of center $p$ and radius $R+R I$

4 For all points $q$ in $\mathcal{C}$, counterclockwise, do

5 If $\operatorname{tmp}(q)=0$ and $D T(q)=a$ then $\quad / /$ new tangency zone

$6 \quad n b_{\text {tang }}=n b_{\text {tang }}+1$

7 Label recursively with the value $n b_{\text {tang }}$ in tmp the 8-neighbours $n_{j}$ 8 of $q$ such that $d\left(p, n_{j}\right) \leqslant R+E T H$ and $D T\left(n_{j}\right)=a$

9 For each tangency zone $\widehat{A B}$ do

10 Compute the maximal distance $d$ between $\widehat{A B}$ and the MCT $[A B]$

11 If $d \leqslant P T H$ then contract the tangency zone to a single point, in the middle of the zone

12 Compute the midpoints of the segments bounded by the extremities of the tangency zones, counterclockwise $\quad / /$ may be empty in the case of a single contracted zone

13 Insert these points as $\alpha$-points in $C A$

14 If $n b_{\text {tang }} \geqslant 3$ then insert $p$ as $\beta$-point in $C A \quad / /$ at least three MCTs

\section{Results and Discussion}

This section deals with the analysis of the chordal axis (CA) produced by our algorithm. We present the CA of different objects, and give some results in terms of shape description. We analyze the influence of the chosen chamfer norm, as well as the threshold values, on the geometry of the CA. An application to shape decomposition is presented; then a connection with the medial axis (MA) is proposed. Finally we have a look at the complexity of the algorithms. 


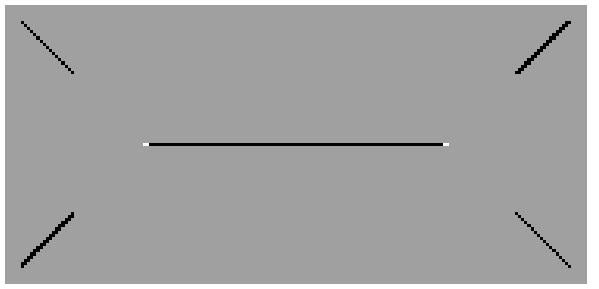

(a)

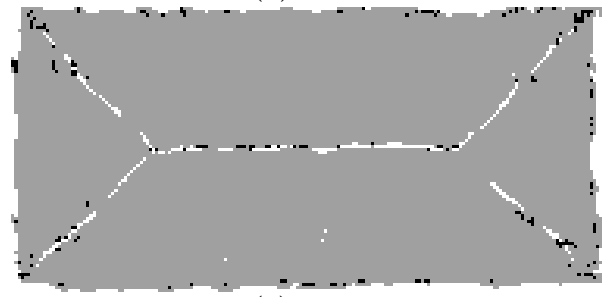

(c)

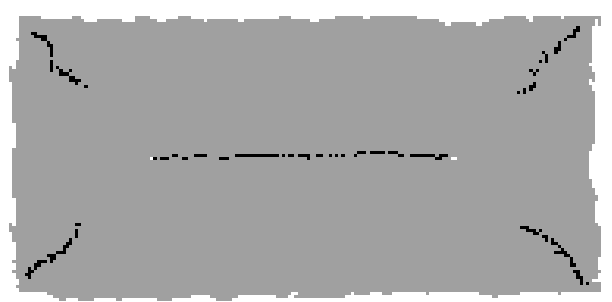

(b)

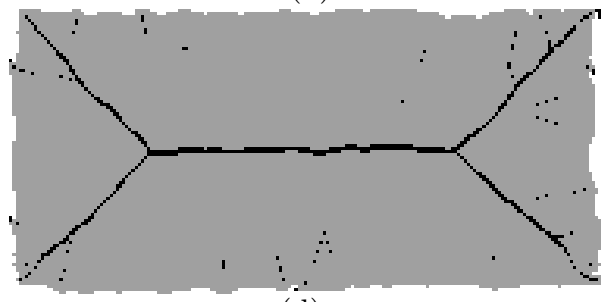

(d)

Fig. 7. CA with thresholds on a rectangle (a) and a noisy rectangle (b); CA without thresholds (c); MA (d). In (a), (b), (c) : $\alpha$-points in black and $\beta$-points in white.

\subsection{Characteristics of the Chordal Axis}

Fig. 7 shows in (a), (b) and (c) the CA of rectangular shapes produced by our algorithm, together with the MA (d), using the chamfer norm $d_{\langle 5,7,11\rangle}$. The input rectangle (in grey) in (b), (c) and (d) has a noisy boundary. In (a), (b), (c), black points are $\alpha$-points, i.e. midpoints of maximal chords of tangency, while white points are $\beta$-points, i.e. centers of maximal ball having at least three MCTs (the CA is composed of both $\alpha$ and $\beta$ points). The CA in (b) is computed using the thresholds method described at Section 3, while (c) is generated without any threshold. The CA in (a) is coherent with the definition of the CA in the continuous plane (see Fig. 1). The differences between (b) and (c) point out the importance of the thresholds. On (b) the extension threshold ETH (set to 10 for this example) erases parasitic $\alpha$-points near the border of the shape; the point threshold PTH (here at 4 pixels) avoids the apparition of superfluous $\beta$-points; the increment threshold $R I$ (set to 1 ) ensures the presence of $\alpha$-points in the middle of the object. Compared to the MA (d), the CA contains less points, is free of parasitic points, and is less connected. We also observe a slight deviation between the MA and the CA in the tips of branches of the shape, because $\alpha$ points may be quite far from centers of maximal balls.

The influence of the chamfer norm on the CA is illustrated in Fig. 8 , When using $d_{4}$ (a) or $d_{8}$ (b), we observe three annoying phenomenons with respect to shape description:

- There are too many $\beta$-points. This is because the distances $d_{4}$ and $d_{8}$ badly approximate the Euclidean distance (their balls are squares).

- In some places of the middle of the branches of the shape, there is a lack of points in the CA. This is also due to the shape of the balls of $d_{4}$ and 


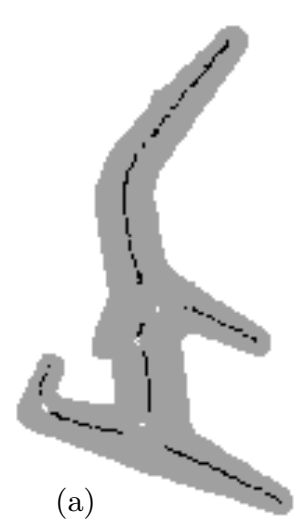

(a)

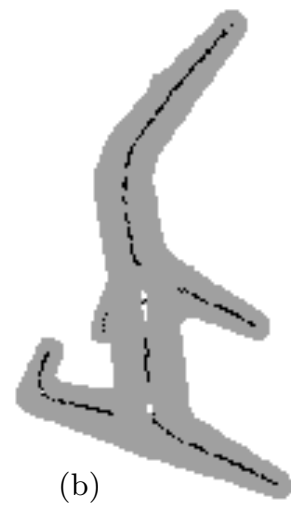

(b)

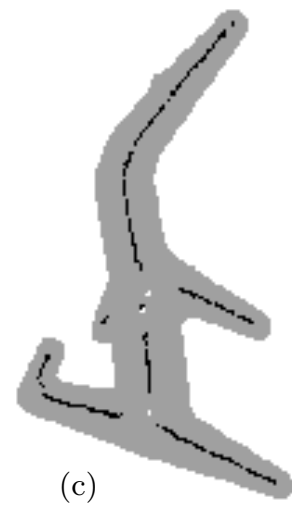

(c)

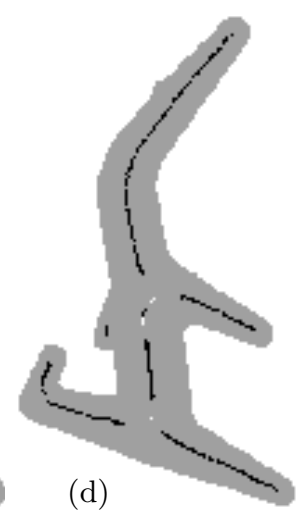

(d)

Fig. 8. CA with $d_{4}(\mathrm{a}), d_{8}(\mathrm{~b}), d_{\langle 3,4\rangle}(\mathrm{c}), d_{\langle 5,7,11\rangle}$ (d). $\alpha$-points appear in black, $\beta$-points in white.

\begin{tabular}{|c|l|l|}
\hline Optimal value & \multicolumn{1}{|c|}{ If less than optimal } & \multicolumn{1}{|c|}{ If greater than optimal } \\
\hline $1 \leqslant R I \leqslant a$ & incomplete CA (see Section [3.3) & $\begin{array}{l}\text { increase of the number of tan- } \\
\text { gency zones } \Rightarrow \text { too many MCTs }\end{array}$ \\
\hline$a \leqslant E T H \leqslant 3 a$ & $\begin{array}{l}\text { parasitic MCTs along the bound- } \\
\text { ary of the shape (see Section [3.2) }\end{array}$ & $\begin{array}{l}\text { rough detail level, lack of MCTs } \\
\text { in thick branches }\end{array}$ \\
\hline $3 \leqslant P T H \leqslant 6$ & $\begin{array}{l}\text { parasitic MCTs along the bound- } \\
\text { ary of the shape (see Section 3.1) }\end{array}$ & $\begin{array}{l}\text { lack of MCTs in maximal balls } \\
\text { having a single tangency zone }\end{array}$ \\
\hline
\end{tabular}

Fig. 9. Influence of threshold values ( $a$ is the weight of the first vector of the chamfer mask)

$d_{8}$, which tends to create MCTs whose extremities are often located in the vertices of the squares. In certain local areas, many midpoints of chords may overlap.

- There is a strong anisotropy of the CA.

These features considerably attenuate when choosing $d_{\langle 3,4\rangle}(\mathrm{c})$ and $d_{\langle 5,7,11\rangle}(\mathrm{d})$; with $d_{\langle 5,7,11\rangle}$ we obtain the best results (the average approximation error compared to $d_{E}$ is only about $2 \%$ ).

An important characteristic of the $\mathrm{CA}$ concerns the localization of its two different kinds of points:

- the $\alpha$-points are located in the branches of the object, and are equidistant from each side of their branch;

- the $\beta$-points are located at the center of branching zones of the shape.

The choice of optimal values of the thresholds, for which the CA describes the shape as well as possible, has been experimentally determined by tests on different objects (with various branching zones and widths). Recommanded values and problems resulting from bad values are listed in Fig. 9. 


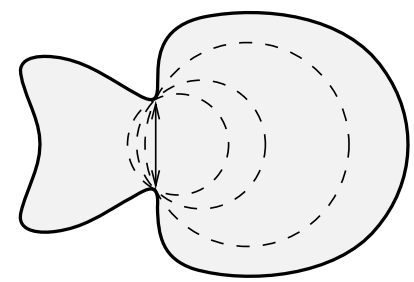

Fig. 10. Maximal balls yielding overlapping MCTs
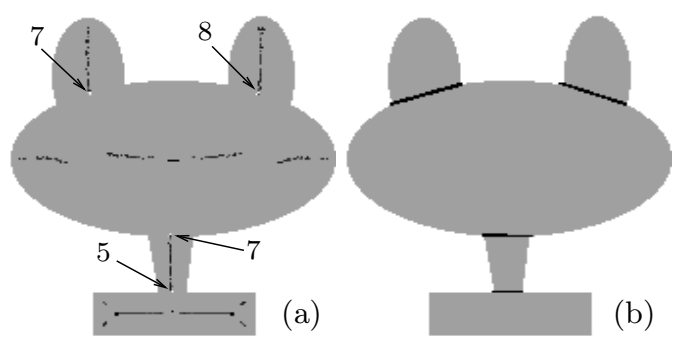

Fig. 11. CA in black and points of high concentration in white (with values) (a); cut chords (in black) (b)
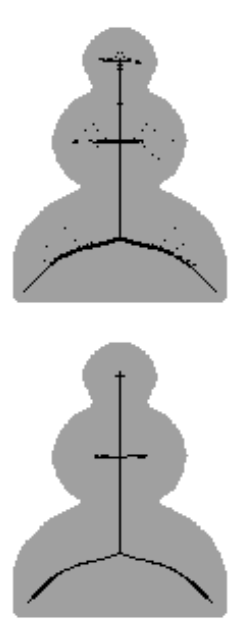
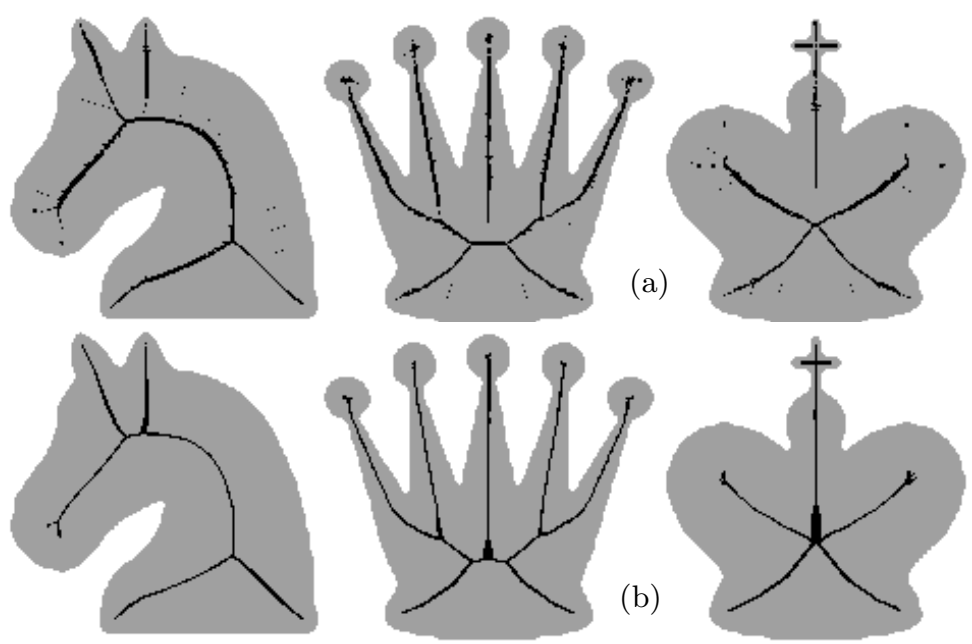

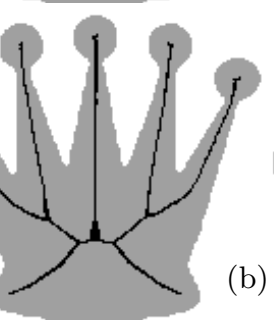

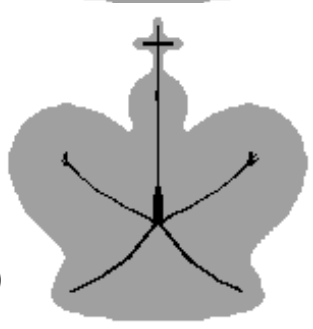

Fig. 12. MA with $d_{\langle 5,7,11\rangle}$ (a); $\mathcal{C}$-CA with $d_{\langle 5,7,11\rangle}, R I=1, E T H=10, P T H=4$ (b)

\subsection{Application to Shape Decomposition}

The CA has an interesting characteristic: points of the axis may be superposed, as shown in Fig. 10. The three maximal balls which are drawn have maximal chords of tangency which overlap each other in the discrete space. We notice that the local concentration of the $\alpha$-points of the CA is high in the zones where the object presents narrowings.

An interesting idea consists in counting, for each point of the image, the number of points of the CA (during computation). We are then able to study the concentration of points of the axis. Points of high concentration are represented in white in Fig.11(a). If for a given point of the image, the number of overlapping points of the $\mathrm{CA}$ is greater than a certain value, denoted cut threshold, then we consider the corresponding MCT as a cut chord for shape decomposition. Each 
cut chord splits the shape into two distinct parts. E.g. in Fig. 11] (b), the cut threshold is set to 5 .

Classic methods of shape decomposition are based upon the reckoning of a skeleton, which must be thin and connected, and the study of gradient along this skeleton. The main advantage of our method is that it does not previously require the creation of a skeleton. The values of local concentrations of points of the $\mathrm{CA}$ are directly calculated during the CA extraction.

\subsection{Connections Between the Chordal Axis and the Medial Axis}

We call $\mathcal{C}$-Chordal Axis (or $\mathcal{C}-C A$ ) of a shape $\mathcal{S}$, the set of centers of all balls included in $\mathcal{S}$ which admit at least a maximal chord of tangency. In $\mathbb{R}^{2}$ the $\mathcal{C}$-CA is exactly the MA, because a ball is maximal in $\mathcal{S}$ if and only if it has at least two points of tangency with the boundary of $\mathcal{S}$ (see [2]). This property is not true in the discrete plane, however our threshold techniques allow the $\mathcal{C}$-CA to approximate the MA while filtering it. We adapt our algorithm of generation of the CA to compute the set of centers of balls inside $\mathcal{S}$ which have at least one MCT. Fig. 12 shows the MA and $\mathcal{C}$-CA of different shapes, using $d_{\langle 5,7,11\rangle}$ and standard thresholds. Notice that both axis have a majority of common points, nevertheless there are significant differences. The $\mathcal{C}$-CA is much more connected than the MA (note that MA can be filtered by post-processing to achieve connectedness, see for instance [13]). Furthermore the $\mathcal{C}$-CA contains much less isolated parasitic points, thanks to the sequence of thresholds which play the role of noise filter when constructing the $\mathcal{C}$-CA. The $\mathcal{C}-\mathrm{CA}$ is not yet an ideal filtering of the MA: it is not guarantied to be connected, and may also be relatively thick in some configurations. A further study of the influence of the threshold values should be realized.

\subsection{Complexity of the Algorithms}

Two preliminary algorithms operate before the very generation of the CA. The first one is the distance transformation algorithm, which computes the DT of the object in time $O\left(m . L^{2}\right)$ for an image of side length $L$ and a chamfer mask having $m$ weightings. The second one is the MA extraction algorithm, whose complexity is $O\left(k \cdot L^{2}\right)$ ( $k$ being a constant close to $m$, see [7]). The CA is then produced from the MA: for each point of the MA, the boundary of a maximal ball is checked. A ball of radius $R$ pixels has about $2 \Pi . R$ boundary points, the examination of the boundary of the ball takes $O(R), R$ being smaller than $L / 2$. Therefore the overall complexity is $O\left(m . L^{2}+n_{M A} . L\right)$, with $n_{M A}$ being the number of points of the MA. However these points represent only a little portion of the points of the shape; in practice we observe that the time complexity is linear in the number of points of the image.

\section{Conclusion}

In this paper, we have adapted Prasad's continuous definition of the chordal axis (CA) [1] in the discrete plane $\mathbb{Z}^{2}$. We have proposed to extract it on a distance 
transform (DT) in the case of chamfer norms, which allows fast extraction of medial axis (MA) 7]. We have introduced three quantities, namely point threshold, extension threshold and radius increment, in the purpose of discretizing the tangency of discrete balls to the boundary of a shape, and properly characterizing maximal chords of tangency (MCT). Then our algorithm extracts the MCTs in near linear time. Changing thresholds enables to filter the noise in shapes, and concentration of CA points allows shape decomposition. Comparison between discrete CA and MA is achieved, which shows a set of new properties for shape description. Future work concerns local detection on DT, study of the reversibility degree, and extension in $3 \mathrm{D}$.

\section{References}

1. Prasad, L.: Morphological analysis of shapes. CNLS Newsletter 139 (1997)

2. Blum, H.: A transformation for extracting new descriptors of shape. In WathenDunn, W., ed.: Models for the Perception of Speech and Visual Form, Cambridge, MIT Press (1967) 362-380

3. Pfaltz, J., Rosenfeld, A.: Computer representation of planar regions by their skeletons. Comm. of ACM 10 (1967) 119-125

4. Montanari, U.: Continuous skeletons from digitized images. Journal of the ACM 16(4) (1969) 534-549

5. Attali, D., Montanvert, A.: Semicontinuous skeletons of $2 \mathrm{~d}$ and $3 \mathrm{~d}$ shapes. In: Aspects of Visual Form Processing, World Scientific, Singapore (1994) 32-41

6. Prasad, L.: Rectification of the chordal axis transform and a new criterion for shape decomposition. In: $11^{\text {th }}$ DGCI, Poitiers (2005)

7. Remy, E., Thiel, E.: Medial Axis for Chamfer Distances: computing LUT and Neighbourhoods in 2D or 3D. Pattern Recognition Letters 23(6) (2002) 649-661

8. Remy, E., Thiel, E.: Exact Medial Axis with Euclidean Distance. Image and Vision Computing 23(2) (2005) 167-175

9. Borgefors, G.: Distance transformations in arbitrary dimensions. Computer Vision, Graphics and Image Processing 27 (1984) 321-345

10. Rosenfeld, A., Pfaltz, J.: Sequential operations in digital picture processing. Journal of ACM 13(4) (1966) 471-494

11. Borgefors, G.: Distance transformations in digital images. Computer Vision, Graphics and Image Processing 34 (1986) 344-371

12. Thiel, E.: Géométrie des distances de chanfrein. HDR, Univ. de la Méditerranée, Aix-Marseille 2 (2001) http://www.lif-sud.univ-mrs.fr/ ${ }^{\sim}$ thiel/hdr.

13. Attali, D., Sanniti di Baja, G., Thiel, E.: Skeleton simplification through non significant branch removal. Image Processing and Communications 3(3-4) (1997) $63-72$ 